\title{
Primary Mediastinal Seminomas: Evidence of Single and Multiple KIT Mutations
}

\author{
Ronald M. Przygodzki, Alan E. Hubbs, Feng-Qi Zhao, and Timothy J. O’Leary \\ Armed Forces Institute of Pathology, Department of Cellular Pathology and Genetics, Rockville, Maryland
}

SUMMARY: Primary mediastinal seminomas (MS) are rare tumors that are histologically similar to their testicular counterparts. Reports document $K I T$ mutations in gastrointestinal stromal tumors, mastocytosis, and germ cell tumors. Although rare exon 17 mutations have been reported in gonadal seminomas, their mediastinal counterparts have not been studied. To determine whether primary MS harbor KIT mutations, eight formalin-fixed, paraffin-embedded primary MS were microdissected; KIT exons 11 and 17 were sequenced. Four (50\%) of the eight cases demonstrated KIT exon 17 mutations. Two of the cases showed single monoallelic base pair alterations; one of these mutations were silent. The other two cases each demonstrated two monoallelic point mutations. In each case, one of these mutations results in protein sequence alteration, and the other is silent. Sequencing of cloned PCR products showed both the silent and amino acid-altering mutations to be found on the same allele (cis). The codon 816 mutation previously identified in mastocytosis and gonadal germ cell tumors was not observed. Non-neoplastic tissues from these patients did not demonstrate KIT mutations; exon 11 mutations were not seen in either tumors or normal tissues. Only the three cases in which amino acid-altering mutations were observed showed a predominantly cytoplasmic CD177 KIT immunohistochemical staining, whereas the one non-amino acid mutating and all wild-type cases were immunonegative. Our findings demonstrate a unique KIT sequence and expression pattern among MS. KIT sequencing may assist in differentiating primary from metastatic MS. (Lab Invest 2002, 82:1369-1375).

$G$ erm cell tumors account for approximately $20 \%$ of all anterior and superior mediastinal tumors and cysts (Bergh et al, 1978; Moran et al, 1997; Shimosato and Mukai, 1997). The pathogenesis is unclear; it seems that these tumors arise from germ cells that have been misplaced during migration and embedded outside of the gonads along the midline structures (Friedman and Van de Velde, 1981). Mediastinal seminomas (MS) usually present as large, nonencapsulated masses in young adult men. These seminomas are microscopically similar to their testicular counterparts, as is their immunohistochemical profile. Survival for patients with MS has been reported from $50 \%$ to $75 \%$ after 5 years.

The KIT gene encodes a type III transmembrane tyrosine kinase receptor (Fig. 1). KIT mutations are found in mast cell diseases, gastrointestinal stromal tumors, and germ cell tumors (Ernst et al, 1998; Longley et al, 1996; Miettinen and Lasota, 2001; Nagata et al, 1995; Tian et al, 1999); many of these

\section{DOI: 10.1097/01.LAB.0000032410.46986.7B}

Received July 3, 2002.

The opinions and assertions contained herein are the private views of the authors and are not to be construed as official or reflecting the views of the Department of Defense. This work was presented in part at the 2002 United States and Canadian Academy of Pathology Meeting in Chicago, Illinois. This work is supported in part by a grant from the American Registry of Pathology.

Address reprint requests to: Dr. Ronald M. Przygodzki, Armed Forces Institute of Pathology, Department of Cellular Pathology and Genetics, 1413 Research Boulevard, Building 101, Rockville, Maryland 20850. E-mail:przygodz@afip.osd.mil alterations produce constitutive gene activation (Hirota et al, 1998; Nagata et al, 1995; Tian et al, 1999). $\mathrm{KIT}$ is also expressed in several cell types during development, where it regulates primordial germ cell migration, proliferation, and apoptosis during fetal gonadal development (Mauduit et al, 1999). KIT deletions, point mutations, and splicing alterations result in modifications of spermatogenesis.

An exon 17 (codon 816) KIT mutation has been previously identified in testicular germ cell tumors (Tian et al, 1999). We sequenced KIT exons 11 and 17 to determine whether similar mutations occur in MS.

\section{Results}

\section{Clinical Background}

All eight MS cases were men with an average age of 27.7 years and an age range from 18 and 40 years at the time of presentation. Each of these tumors was localized in the anterior mediastinum, and each patient had a negative clinical history for other tumors.

Histologically the tumors were characterized by the cellular proliferation of uniform polygonal to round cells arranged in sheets and nests separated by thin bands of fibroconnective tissue septae found in this tumor type (Fig. 2). The nuclei were centrally located, round to oval, and some had prominent nucleoli. Mitotic figures were rare. Lymphoid cells were present in all of the cases along thin fibrous bands and intermingling with the neoplastic cells. Periodic-acid Schiff stain showed moderate amounts of glycogen within the cytoplasm. Immunohistochemical reactivity with placental-like alkaline phosphatase was seen in 


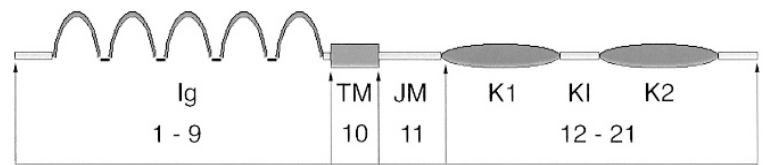

Figure 1.

Cartoon of KIT protein product and corresponding exon regions. Exon 11 of KIT is localized to the juxtamembrane domain (JM), whereas exon 17 is localized to one of two kinase domains (K2). Numbers correspond to exon(s) coding for a given region. $l g=$ immunoglobulin region; $T M=$ transmembrane domain; $J M=$ juxtamembrane domain; $K 1$ and $K 2=$ kinase regions; $K I=$ kinase interspace.

all the cases, whereas $\alpha$-fetoprotein, carcinoembryonic antigen, and $\beta$-human chorionic gonadotrophin were negative in all cases.

\section{KIT Exon 17 Sequence and Cloning Analyses}

KIT exon 17 gene sequence analysis identified mutation(s) in four (50\%) of the eight cases (Table 1). All eight nontumor tissue samples were wildtype. The gastrointestinal stromal tumor and testicular seminoma controls were wildtype.

All six mutations found in the four cases were unique (Figs. 3 and 4). In two cases a single base pair mutations was found (Table 1), whereas in two additional cases two mutations were identified in the tumors. Codon 816 mutations, which have been found previously in germ cell tumors and mastocytosis ( $\mathrm{Na}$ gata et al, 1995; Tian et al, 1999), were not identified in our cases. In the two cases harboring two mutations, the second coding base pair was mutated, producing an amino acid code change from aspartic acid to valine, and lysine to arginine, respectively. The second mutation in these cases was silent, ie, the mutation did not change the coded amino acid. In one of the cases harboring a single base alteration, the coding amino acid change was from asparagine to lysine; in the remaining case, the mutation was silent.

We sequenced cloned exon 17 KIT PCR fragments to determine whether those cases with two point mutations had these alterations on the same DNA strand (in cis orientation) or whether the alterations occurred on different DNA strands (trans orientation). In each case, over 20 colonies were picked for this identification. For each case, more than half of the colonies demonstrated the silent mutations, and another quarter showed both mutations on the same DNA strand (cis mutations). No colonies were found to solely have the amino acid-altering mutations.

The predominant base pair alterations were three $\mathrm{C}: \mathrm{G}>\mathrm{T}: \mathrm{A}$ transition mutations, one A:T $>\mathrm{G}: \mathrm{C}$ transition mutation, one $\mathrm{A}: \mathrm{T}>\mathrm{T}: \mathrm{A}$ transversion mutation, and one $\mathrm{T}: \mathrm{A}>\mathrm{G}: \mathrm{C}$ transversion mutation. Three of four transition mutations produced silent mutations, whereas all transversion mutations altered the amino acid coding sequence. Both of the two-mutation cases (Cases 2 and 4) had a silent transition mutation and amino acid-converting transversion mutation. Both single-mutation cases were of transition type, with one case producing a silent mutation and the other producing an amino acid-converting mutation.

\section{KIT Exon 11 Analysis}

All eight cases successfully amplified both the nontumor and tumor samples for the exon 11 PCR fragment. We did not find any KIT exon 11 gene sequence alteration within the coding and juxtaposed flanking intron sequences. We did not identify any point mutation, deletion, or insertion alterations in any of the nontumor and tumor tissues. The control gastrointestinal stromal tumor had an in-frame deletion, whereas all three control testicular seminomas were wildtype.

\section{CD117a (KIT) Immunostaining}

All eight cases underwent immunohistochemical staining. Three of the four exon 17 KIT mutated cases were positive for immunostaining and included both of the dual-mutant as well as one single-mutant amino acid-altering cases. These three cases revealed diffuse cell membrane and cytoplasmic immunohistochemical staining of the seminomatous component. The intensity of immunostaining within the seminomatous component was, however, varied, with most of the tumor cells aggregates staining moderately, and the admixed somewhat minor component staining intense, imparting a mottled pattern of staining (Fig. 5). The fourth mutated, yet immunostain-negative case is Case 5 (Fig. 3). The mutation in this latter case does not produce an amino acid alteration and therefore is a silent mutation. The intervening lymphocytes were negative in all cases. Positive immunohistochemical controls with gastrointestinal stromal tumor and testicular seminomas revealed typical cytoplasmic/cell membrane and cell membrane staining, respectively (Fig. 5, inset). The negative controls were appropriately negative.

\section{KIT Alteration and Survival}

No significant survival difference was observed between the nonmutated versus either single or dual KIT mutated MS. No significant correlations were found between tumor size, age of onset, or length of disease-free survival and KIT mutation.

\section{Discussion}

Primary MS are rare malignancies that microscopically resemble their gonadal counterparts. They have not been studied extensively with respect to their genetic structure. We undertook this study to identify whether KIT exon 11 and 17 alterations exist within this unique tumor type. We found half of the MS cases to have point mutation(s) in exon 17 of the KIT gene. Half of the mutated cases had a second point mutation in exon 17 in cis, ie, on the same DNA stand. The remaining cases, as well as all of the exon $11 \mathrm{KIT}$ gene cases, had wild-type gene sequences.

Our results contrast with those found by Tian et al (1999) and Nagata et al (1995), who found that germ cell tumors (including seminomas), and mastocytosis, demonstrate point mutation at codon 816 of KIT exon 17. The mutation identified in germ cell tumors is the 


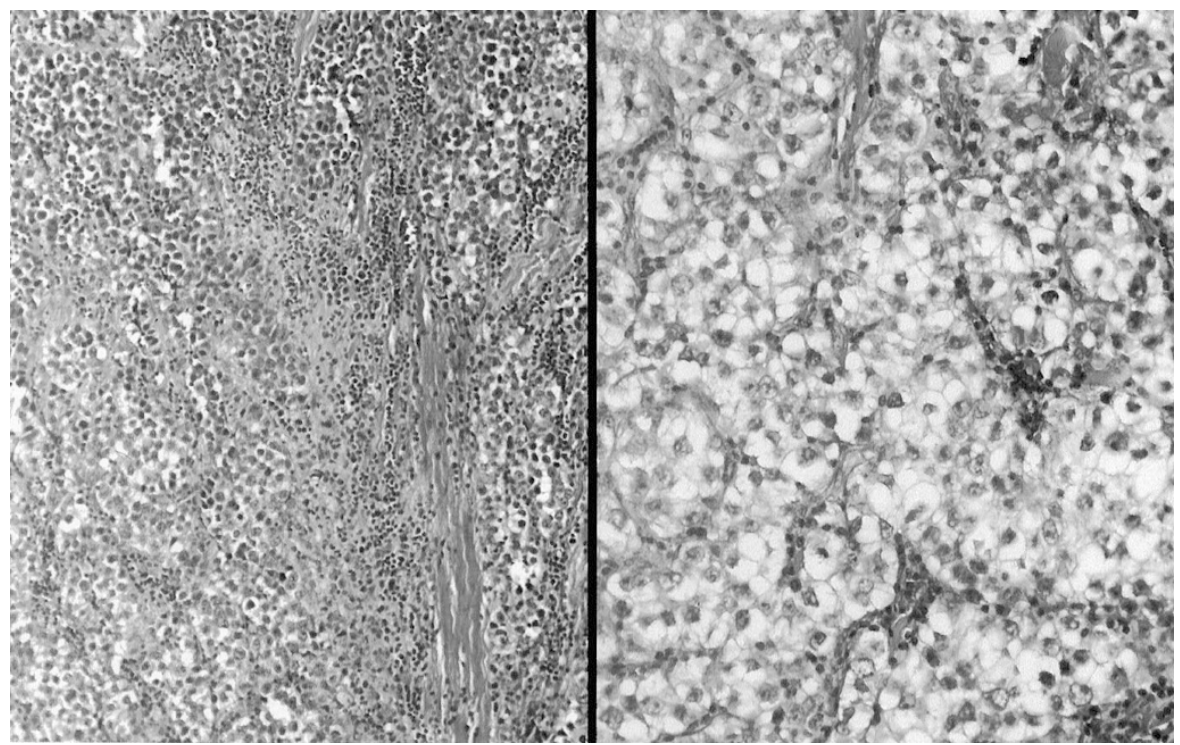

Figure 2.

Histologic examination of mediastinal seminomas (MS). The neoplastic cells are composed of uniform polygonal to round cells arranged in sheets and nests separated by collagenous stroma. Lymphoid cells occur predominantly in juxtaposition to the fibrous bands but also intermingled in between the neoplastic cells. Hematoxylin and eosin stain: left, magnification $\times 100$; right, magnification $\times 200$.

Table 1. MS Gene Mutations Profiles Found Among Individual Cases

\begin{tabular}{ccc}
\hline $\begin{array}{c}\text { Case } \\
\text { no. }\end{array}$ & Sequence change & Codon \\
\hline 2 & ATC $>$ ATT & 798 \\
& GAT $>$ GTT & 820 \\
4 & ATC $>$ ATT & 805 \\
& AAT $>$ AAG & 822 \\
5 & CTC $>$ CTT & 799 \\
7 & AAG $>$ AGG & 818 \\
\hline
\end{tabular}

transversion mutation GAC $>$ CAC, yielding an amino acid change of aspartic acid to histidine. The mutation found in mastocytosis, also a mutation of the transversion type, is a GAC > GTC alteration, resulting in a change of amino acid from aspartic acid to valine. None of the eight cases we analyzed had this mutation. This difference in mutational profile may prove diagnostically useful in some patients.

The occurrence of dual mutations in cis in one quarter of our cases is interesting. We and other investigators have previously identified silent mutations in KIT (Ernst et al, 1998; Miettinen and Lasota, 2001; Nagata et al, 1995; Paquette et al, 1996; Tian et al, 1999). Unfortunately, one study that found both silent and amino acid-altering mutation in a case of mixed dysgerminoma/yolk sac tumor failed to note whether the alteration was in cis or in trans (Tian et al, 1999).

Our cloning and sequencing results suggest that in the tumors demonstrating two mutations, the first mutation was silent and was subsequently followed by a second amino acid-altering mutation. This hypothesis is supported by our findings of a distinct tumor population with a single silent mutation in the up- stream portion of exon 17 , followed by another distinct yet smaller population with both silent and amino acid-altering mutation in cis. None of the dual-mutant cases had a sole population of amino acid-altering mutant changes. No gene alterations were found among the normal adjacent tissue samples of each case.

Previously reported studies identified CD117 cell membrane immunopositivity in seminomas (Izquierdo et al, 1995; Rajpert-De Meyts et al, 1996; Strohmeyer et al, 1995; Tian et al, 1999; Tsuura et al, 1994). Most of these studies focused on seminomas of gonadal origin, with only a few extragonadal cases included. Only the study by Tian and coworkers (1999) included both genomic analysis of KIT and CD117 immunostaining in gonadal seminomas. Our study focused on primary MS and included KIT genomic analysis and CD117 immunostaining. The three testicular seminoma controls in this study were KIT exon 11 and 17 wildtype, which is in agreement with 2 of 23 mutated cases found in Tian's work. Moreover, these controls showed cell membrane immunostaining, which is supportive of the previous studies (Fig. 5, inset). In contrast, we found that MS either did not stain (Fig. 5, left) or had a predominantly cytoplasmic/cell membrane staining pattern of the tumor cells, with significant cell-to-cell heterogeneic "mottling" (Fig. 5, right). Importantly, the negative and positive immunostaining results correlated with both wild-type exon 11 and 17 $K I T$, or wild-type exon 11 and mutant exon $17 \mathrm{KIT}$, respectively. Only the MS cases with mutations producing an amino acid change are cytoplasmic/cell membrane immunopositive for KIT (CD117), whereas the remaining cases were immunonegative.

The immunohistochemical staining pattern found among MS suggests some possible interpretations of the study. The predominantly cytoplasmic staining of 


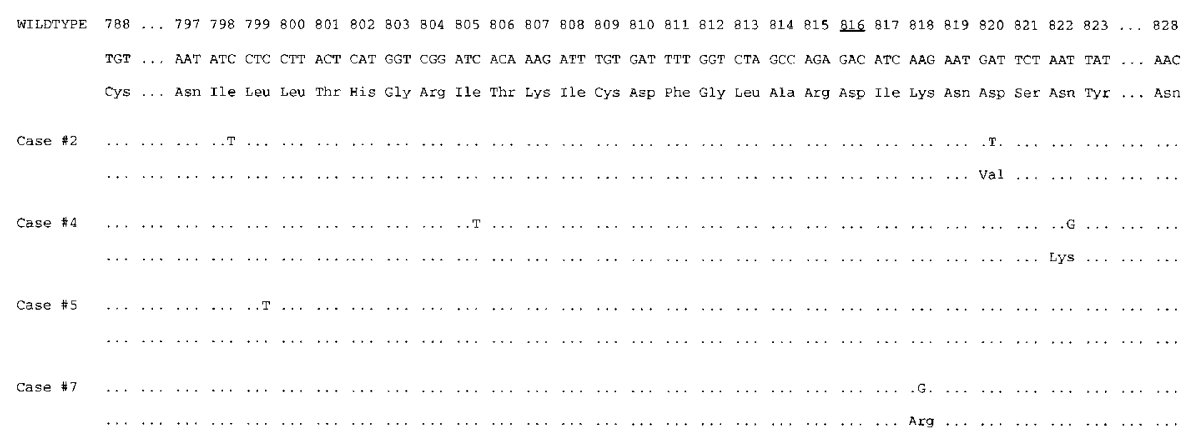

Figure 3.

Exon 17 coding sequence of KIT. A portion of the wild-type sequence of codon 17 is presented. Specific point mutations evident in the MS cases mutated are identified. When a given point mutation produces an amino acid substitution, the corresponding amino acid change is noted. Codon 816 is underlined and is identified as the codon wherein gonadal germ cell tumors are typically mutated.
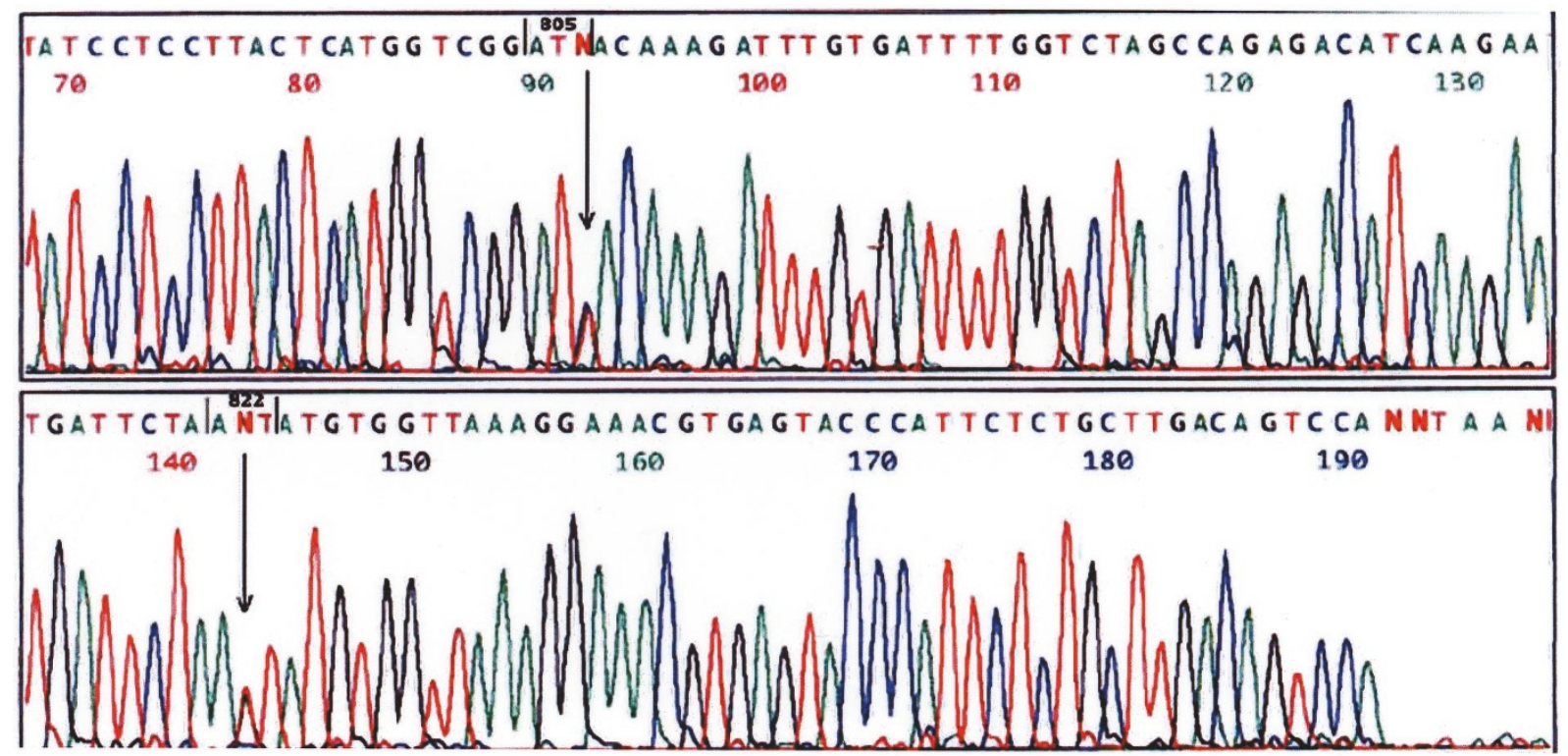

Figure 4.

KIT exon 17 sequence alterations in Case 4. There is evidence of codon 805 silent mutation alteration (ATC > ATT) and amino acid substituting mutation in codon $822\left(\right.$ AAT $^{\text {Asn }}>$ AAG $\left.{ }^{\text {Lys }}\right)$.

KIT may reflect a lack of cell surface expression. KIT is required in germ cell migration during embryogenesis (Mauduit et al, 1999). Thus, the lack of purely membranous KIT expression found in the MS may actually support the reason for halt in cell migration to the gonads. Alternatively, this effective lack of KIT expression may in turn lead to abnormal growth regulation and possible malignant transformation, thereby producing a more aggressive tumor (Strohmeyer et al, 1995). Finally, the source of antibodies used in the previously identified studies was different from the one used in this study, which is currently the one used most commonly.

The exon 17 region of KIT corresponds to one of two kinase domains in this receptor tyrosine kinase. Investigators have identified several key codons in this region linked with kinase activity. These include KITmediated mitogenesis and survival associated with codon 821, an active site at codon 792, an autophosphorylation site at codon 823 , and codon 816 muta- tion associated with gene activation (Nagata et al, 1995; Serve et al, 1995; Tian et al, 1999). Taken together with the cloning and sequencing results, the findings might suggest alterations in KIT activity; however, we have not validated this finding experimentally. The immunohistochemical findings suggest KIT accumulation in the cytoplasm. The lack of predominantly cell membrane KIT immunostaining, but rather predominantly cytoplasmic staining, or no staining may reflect a unique finding among primary MS that seems to be linked with either KIT exon 17 mutation or wild-type exon 11 and 17 KIT, respectively.

In summary, we have identified novel single and double mutations in exon 17 of KIT in MS. The double mutations identified are in a cis configuration. Cloning and sequencing KIT exon 17 suggests that in some cases an initial silent mutation is followed by a second cis mutation giving rise to amino acid alteration and KIT activation. Most of the mutations observed are of 

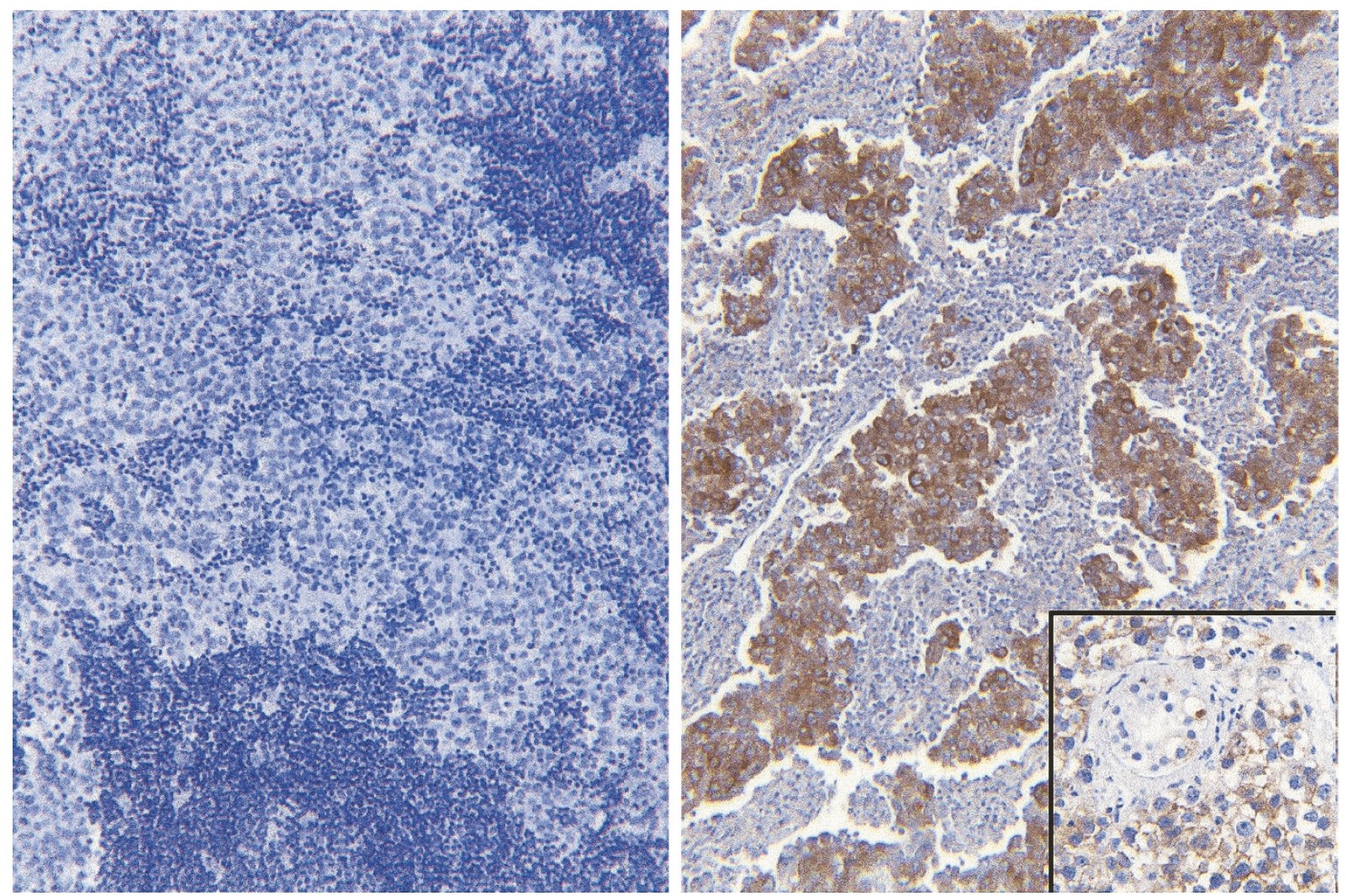

\section{Figure 5.}

CD117a (KIT, clone C-19) immunohistochemical staining of MS. Left, an MS case that is KIT exon 11 and 17 wildtype and CD117a immunostain negative. Right, a KIT exon 17 with two mutations. The immunopositivity is cytoplasmic and within the tumor cells. The intensity varies and brings forth a "mottled" appearance, with the majority of the tumor cells moderately positive and some tumor cell nests staining intensely. The inset demonstrates the control testicular seminoma with typical cell membrane immunostaining. Both panels, magnification $\times 100$; inset, magnification $\times 200$.

the C:G > T:A transition type; however, the amino acid-altering mutations were transversion mutations. KIT exon 11 mutations were not identified in any of the MS samples. CD117a immunostaining of MS reveals a markedly different staining pattern from gonadal seminomas, in that either predominantly cytoplasmic or no immunostaining is usually found. Cytoplasmic immunostaining is found among cases with amino acidaltering exon 17 KIT mutations, whereas no staining is found among exon 11 and 17 wild-type cases or non-amino acid altering mutation(s) in KIT exon 17. Finally, the observation of different mutations in MS and testicular germ cell tumors of the gonads may prove useful diagnostically.

\section{Materials and Methods}

\section{Case Sampling}

Eight formalin-fixed, paraffin-embedded primary MS were obtained from the archives of the Armed Forces Institute of Pathology. Review of the case histories showed none of these patients to have a history of gonadal tumor-type malignancy(ies) elsewhere. Histologic slides were reviewed using standardized histopathologic criteria (Mostofi et al, 1988). A representative formalin-fixed, paraffin-embedded tissue block from each case was selected for immunohistochemi- cal and gene sequence analysis of each case. Immunohistochemical staining for placental-like alkaline phosphatase, $\beta$-human chorionic gonadotrophin, $\alpha$-fetoprotein, and carcinoembryonic antigen was used to confirm the impressions based on hematoxylin and eosin-stained material. Follow-up on the eight cases ranged from 1 to 17 years.

\section{Tissue Sampling and Molecular Methodologies}

Four $5-\mu \mathrm{m}$ thick histologic sections were cut from each representative tissue block. The first section was stained with hematoxylin and eosin and was reviewed to guide microdissection. One slide for KIT (CD117a) immunohistochemical staining was deparaffinized and pretreated with microwave antigen retrieval using 10 mм citric acid (pH 6.0). Immunohistochemical staining with a 1:1600 dilution of a polyclonal antibody to KIT protein (CD117a, clone C-19; Santa Cruz Biotechnology, Santa Cruz, California) was performed using the avidin-biotin-peroxidase complex method (Hsu et al, 1981). This antibody clone has been epitope mapped to within the carboxy terminal domain of KIT p145 (CD117) of human origin. Thymus and normal lung provided a negative immunostaining control, and a gastrointestinal stromal-smooth muscle tumor and three testicular seminomas were used as positive 
immunostaining controls. Intensity of immunostaining was scored as non-staining; weak; moderate; and intense. Distribution of staining was scored as cell membrane, cytoplasmic, or both.

Two unstained sections from each of the eight cases, and all positive controls, underwent deparaffinization and rehydration using methods previously described (Finkelstein et al, 1996; Przygodzki et al, 2001a, 2001b). Normal and tumor tissues were sampled independently. Care was taken to avoid sampling an excess of lymphoid nontumor tissue to minimize the effect of dilution. We, as well as others, have previously identified that sequence analysis is feasible in samples that have undergone dilution as low as $20 \%$ mutant $/ 80 \%$ wild-type sequence (Finkelstein et al, 1993). All normal and tumor samplings were incubated overnight at $55^{\circ} \mathrm{C}$ in lysis buffer (Finkelstein et al, 1996; Przygodzki et al, 2001a, 2001b). The proteinase $\mathrm{K}$ was subsequently inactivated by a 10 -minute incubation at $95^{\circ} \mathrm{C}$. The resulting lysate was spun in a microcentrifuge for 5 minutes to pellet the debris, and then stored at $-20^{\circ} \mathrm{C}$.

Amplification of exons 11 and 17 of the KIT gene was performed by PCR analysis using primers flanking each exon (Ernst et al, 1998; Tian et al, 1999). Reactions were carried out in a total volume of $50 \mu \mathrm{L}$ in the presence of $2.5 \mathrm{~mm}$ magnesium chloride; $10 \mathrm{~mm}$ Tris-hydrochloric acid, $\mathrm{pH} 8.3 ; 50 \mathrm{~mm}$ potassium chloride; $0.2 \mathrm{~mm}$ each deoxynucleoside triphosphate; $1 \mu \mathrm{M}$ each primer; and 1.5 U Amplitaq Gold (Perkin-Elmer Applied Biosystems, Foster City, California). Two microliters of the genomic DNA extract was used as template for the reaction. PCR amplification was performed on a 9600 Perkin-Elmer Thermocycler (PerkinElmer Applied Biosystems) using the following conditions for both primer pair sets: $95^{\circ} \mathrm{C}$ for 10 minutes of initial heat denaturation and 40 cycles of $94^{\circ} \mathrm{C}(45$ seconds), $53^{\circ} \mathrm{C}$ (30 seconds), and $72^{\circ} \mathrm{C}$ (30 seconds).

Amplified PCR products were separated on a $2 \%$ agarose gel (SeaKem; FMC Corporation, Rockland, Maine) containing ethidium bromide. PCR products were then cut from the agarose gel and extracted using Silica Beads (GeneClean; Bio101, La Jolla, California). Both KIT exon 11- and 17-purified PCR product subsequently underwent sequence analysis, whereas only exon 17-purified PCR products underwent cloning.

Sequencing of the exon 11 and 17 KIT PCR products was performed using the Perkin-Elmer Big-Dye Terminator cycle-sequencing kit on a 377 automated sequencer (Perkin-Elmer Applied Biosystems). Sequence abnormalities were confirmed by a second amplification and sequencing using the reverse primer.

KIT exon 17 PCR fragments were also cloned into a pCR 4-TOPO vector and transformed into TOP10 One-Shot cells using the TOPO TA cloning kit for sequencing following the manufacturer's instructions (Invitrogen, Carlsbad, California). From 10 to 30 colonies for each transformation were picked for colony PCR using M13 forward and reverse primers. This $\mathrm{PCR}$ reaction was used to assess whether primary
PCR inserts were incorporated. If insert was found to be present, a sterile toothpick was used to stab the PCR product band and was subsequently stabbed into sequencing mix using the Big-Dye Terminator cycle-sequencing kit with forward or reverse M13 primers for each sequence analysis.

\section{Acknowledgement}

We thank Dr. Robert Becker for his excellent assistance in the photographing of the cases.

\section{References}

Bergh NP, Gatzinsky P, Larsson S, Lundin P, and Ridell B (1978). Tumors of the thymus and thymic region: III. Clinicopathological studies on teratomas and tumors of germ cell type. Ann Thorac Surg 25:107-111.

Ernst SI, Hubbs AE, Przygodzki RM, Emory TS, Sobin LH, and O'Leary TJ (1998). KIT mutation portends poor prognosis in gastrointestinal stromal/smooth muscle tumors. Lab Invest 78:1633-1636.

Finkelstein SD, Przygodzki R, Pricolo VE, Sakallah SA, Swalsky PA, Bakker A, Lanning R, Bland KI, and Cooper DL (1996). Prediction of biologic aggressiveness in colorectal cancer by $\mathrm{p} 53 / \mathrm{K}$-ras-2 topographic genotyping. Mol Diagn $1: 5-28$.

Finkelstein SD, Sayegh R, Christensen S, and Swalsky PA (1993). Genotypic classification of colorectal adenocarcinoma: Biologic behavior correlates with K-ras-2 mutation type. Cancer 71:3827-3838.

Friedman NB and Van de Velde RL (1981). Germ cell tumors in man, pleiotropic mice, and continuity of germplasm and somatoplasm. Hum Pathol 12:772-776.

Hirota S, Isozaki K, Moriyama Y, Hashimoto K, Nishida T, Ishiguro S, Kawano K, Hanada M, Kurata A, Takeda M, Muhammad Tunio G, Matsuzawa $Y$, Kanakura $Y$, Shinomura $Y$, and Kitamura $Y$ (1998). Gain-of-function mutations of c-kit in human gastrointestinal stromal tumors. Science 279:577580.

Hsu SM, Raine L, and Fanger H (1981). The use of antiavidin antibody and avidin-biotin-peroxidase complex in immunoperoxidase technics. Am J Clin Pathol 75:816-821.

Izquierdo MA, Van der Valk P, Van Ark-Otte J, Rubio G, Germa-Lluch JR, Ueda R, Scheper RJ, Takahashi T, and Giaccone G (1995). Differential expression of the c-kit protooncogene in germ cell tumours. J Pathol 177:253-258.

Longley BJ, Tyrrell L, Lu SZ, Ma YS, Langley K, Ding TG, Duffy T, Jacobs P, Tang LH, and Modlin I (1996). Somatic c-KIT activating mutation in urticaria pigmentosa and aggressive mastocytosis: Establishment of clonality in a human mast cell neoplasm. Nat Genet 12:312-314.

Mauduit C, Hamamah S, and Benahmed M (1999). Stem cell factor/c-kit system in spermatogenesis. Hum Reprod Update 5:535-545.

Miettinen M and Lasota J (2001). Gastrointestinal stromal tumors: Definition, clinical, histological, immunohistochemical, and molecular genetic features and differential diagnosis. Virchows Arch 438:1-12. 
Moran CA, Suster S, Przygodzki RM, and Koss MN (1997). Primary germ cell tumors of the mediastinum: II. Mediastinal seminomas-a clinicopathologic and immunohistochemical study of 120 cases. Cancer 80:691-698.

Mostofi FK, Sesterhenn IA, and Davis CJ Jr (1988). Developments in histopathology of testicular germ cell tumors. Semin Urol 6:171-188.

Nagata $\mathrm{H}$, Worobec AS, Oh CK, Chowdhury BA, Tannenbaum S, Suzuki Y, and Metcalfe DD (1995). Identification of a point mutation in the catalytic domain of the protooncogene c-kit in peripheral blood mononuclear cells of patients who have mastocytosis with an associated hematologic disorder. Proc Natl Acad Sci USA 92:10560-10564.

Paquette RL, Hsu NC, and Koeffler HP (1996). Analysis of c-kit gene integrity in aplastic anemia. Blood Cells Mol Dis 22:159-168.

Przygodzki RM, Goodman ZD, Rabin L, Centeno JA, Liu Y, Hubbs AE, and O'Leary TJ (2001a). Hemochromatosis (HFE) gene sequence analysis of formalin-fixed, paraffinembedded liver biopsy specimens. Mol Diagn 6:227-232.

Przygodzki RM, Koss MN, and O'Leary TJ (2001b). Pleomorphic (giant and/or spindle cell) carcinoma of lung shows a high percentage of variant CYP1A1*2. Mol Diagn 6:109-115.

Rajpert-De Meyts E, Kvist M, and Skakkebaek NE (1996). Heterogeneity of expression of immunohistochemical tumour markers in testicular carcinoma in situ: Pathogenetic relevance. Virchows Arch 428:133-139.
Serve H, Yee NS, Stella G, Sepp-Lorenzino L, Tan JC, and Besmer P (1995). Differential roles of PI3-kinase and Kit tyrosine 821 in Kit receptor-mediated proliferation, survival and cell adhesion in mast cells. EMBO J 14:473-483.

Shimosato $Y$ and Mukai K (1997). Tumors of the mediastinum. In: J Rosai and LH Sobin, editors. Atlas of tumor pathology. Washington, DC: Armed Forces Institute of Pathology, 183-206.

Strohmeyer T, Reese D, Press M, Ackermann R, Hartmann M, and Slamon D (1995). Expression of the c-kit protooncogene and its ligand stem cell factor (SCF) in normal and malignant human testicular tissue. J Urol 153:511-515.

Tian Q, Frierson HF Jr, Krystal GW, and Moskaluk CA (1999). Activating c-kit gene mutations in human germ cell tumors. Am J Pathol 154:1643-1647.

Tsuura Y, Hiraki H, Watanabe K, Igarashi S, Shimamura K, Fukuda T, Suzuki T, and Seito T (1994). Preferential localization of c-kit product in tissue mast cells, basal cells of skin, epithelial cells of breast, small cell lung carcinoma and seminoma/dysgerminoma in human: Immunohistochemical study on formalin-fixed, paraffin-embedded tissues. Virchows Arch 424:135-141. 